\title{
Type 1 diabetes mellitus (T1DM) in toddlers and schoolchildren in Najran
}

\section{region, Southwestern Saudi Arabia-Correlation with osteocalcin and vitamin D}

Mohammed Ayed Huneif1, Elhashimi Eltayb Hassan Homada², Omar Eltayeb Fadlelseed², Hassan

Gumaa Mustafa Hamid², and Mohammed Helmy Faris Shalayel ${ }^{3}$

1. Department of Pediatrics, College of Medicine, Najran University, Najran, Saudi Arabia

2. College of Medical Applied Sciences, Najran University, Najran, Saudi Arabia

3. Department of Biochemistry, College of Medicine, Najran University, Najran, Saudi Arabia

\section{RESEARCH}

Please cite this paper as: Huneif MA, Homada EEH, Fadlelseed OE, Hamid HGM, Shalayel MHF. Type 1 diabetes mellitus (T1DM) in toddlers and schoolchildren in Najran region, Southwestern Saudi Arabia-Correlation with osteocalcin and vitamin D. AMJ 2017;10(12):981-988. https://doi.org/10.21767/AMJ.2017.3184

Corresponding Author:

Mohammed Helmy Faris Shalayel

Department of Biochemistry, College of Medicine

Najran University, Najran, Saudi Arabia

Email:drmhfs@hotmail.com

\section{ABSTRACT}

\section{Background}

There is an ongoing interest in the relationship between vitamin $D$ status and diabetes control and complications. However, data from Saudi Arabia are limited. Human studies demonstrated the positive association between insulin secretion, insulin sensitivity and serum levels of osteocalcin and vitamin D.

\section{Aims}

This study aimed to explore the possible role of circulating osteocalcin in the pathogenesis of T1DM and its correlation with fasting plasma glucose (FPG) and vitamin D levels in Saudi Arabia children with T1DM.

\section{Methods}

This cross-sectional observational hospital based- case control study that included 132 Saudi children clinically diagnosed as type 1 diabetes mellitus and 72 apparently healthy children.

\section{Results}

There were statistically weak negative correlations for FPG with UCOC and TOC in diabetic children ( $r=-0.294, p<0.01$ and $-0.358, p<0.0001$ respectively). A moderately significant positive correlation between FPG and $\mathrm{BMI}$ and a significantly negative correlation between 25(OH)D3 and FPG $(r=-0.62, p<0.0001)$ were found. Moreover, there was a moderately significant positive correlation between levels of vitamin D and UCOC in healthy control group ( $r=0.457$, $p<0.0001)$. Such significance was not present in diabetic group ( $r=-0.077, p<0.38)$.

\section{Conclusion}

Levels of 25(OH)D3, TOC, and UCOC were significantly diminished in children with T1DM suggesting the bidirectional influence of $\beta$ cells on vitamin $D$ and osteocalcin and vice versa. The reverse correlation between UCOC and HbA1c may indicate the probable prognostic value of UCOC as the lower UCOC, the worse glycaemic control in diabetic children.

\section{Key Words}

Type 1 diabetes mellitus (T1DM), under-carboxylated osteocalcin (UCOC), 25(OH)D3, glycated haemoglobin (HbA1c), body mass index (BMI)

\section{What this study adds:}

\section{What is known about this subject?}

The emergence of diabetes mellitus as a global public health problem in children and adolescents is due to the widespread obesity and pronounced lifestyle changes.

\section{What new information is offered in this study?}

This study suggests a bidirectional influence of $\beta$ cells on vitamin $D$ and osteocalcin and vice versa. The reverse correlation between $\mathrm{UCOC}$ and $\mathrm{HbA} 1 \mathrm{C}$ may indicate the probable prognostic value of UCOC. 
3. What are the implications for research, policy, or practice?

This study reported a significant positive correlation between levels of 25(OH)D3 with UCOC and 25(OH)D3 with FPG in healthy control group. Therefore, convenient vitamin D supplementation for children could help to counter the growing trend in the pathogenesis of T1DM.

\section{Background}

Diabetes is one of the fastest-growing chronic diseases worldwide. ${ }^{1}$ Type 1 diabetes mellitus (T1DM) is a chronic autoimmune disorder caused by destruction of beta cells of the pancreas. ${ }^{2}$ The emergence of diabetes mellitus as a global public health problem in children and adolescents is due to the widespread obesity and pronounced lifestyle changes. ${ }^{3}$ Patients with T1DM are at high risk of developing other autoimmune diseases, such as celiac disease and autoimmune thyroiditis. ${ }^{4}$

T1DM can lead to numerous complications in paediatric patients, with the most common acute complications including hypoglycaemia, and diabetes ketoacidosis (DKA). Long-term complications in childhood include retinopathy, nephropathy, and dyslipidemia. ${ }^{5}$ Notwithstanding, risk factors for uncontrolled hyperglycaemia and levels of glycaemic control are not well studied in Saudi children with type 1 diabetes mellitus. ${ }^{6}$ Impaired fasting glucose (IFG) and diabetes mellitus are highly prevalent disorders in Saudi Arabia with the plurality of the patients being unaware of their conditions, which necessitates imperious programmes for early detection, treatment and prevention. ${ }^{7}$

Approximately, 75 per cent of all cases of type 1 diabetes mellitus are diagnosed in individuals $<18$ years of age. ${ }^{8}$ There is an ongoing concern with regard to the correlation between vitamin $D$ status and diabetes control and complications. However, data from Saudi Arabia are limited. In a recent study, vitamin $D$ deficiency was highly prevalent and was found to be associated with frequency of hypoglycaemic episodes and with adverse cardiometabolic control. ${ }^{9}$ Osteocalcin (OC) is a bone-specific protein secreted by osteoblasts and often used as a bone formation biomarker. OC undergoes post-translational carboxylation to yield carboxylated osteocalcin and under-carboxylated osteocalcin (UCOC) molecules. ${ }^{10}$ As far as bone mass is concerned, in adult patients with type 1 diabetes a moderately reduced bone mineral density has been shown in both appendicular and axial skeleton. However, this issue is not well studied in children. ${ }^{11,12}$ The vitamin D endocrine system is involved in many biological processes including regulation of cell proliferation and cell differentiation, bone metabolism, and modulation of immune responses. ${ }^{13}$ While there is increasing evidence that Vitamin D deficiency may be a risk factor for diabetes, over-all evidence regarding vitamin D levels and diabetes mellitus is contradictory, requiring further studies. ${ }^{14}$

Our study aimed to explore the possible role of circulating osteocalcin in the pathogenesis of diabetes mellitus and its correlation with fasting plasma glucose and vitamin D levels in Saudi Arabia children with T1DM.

\section{Method}

This is a cross-sectional observational hospital based- case control study that was conducted in Najran University Hospital and King Khalid Hospital (Najran, Saudi Arabia) from March 2015 till August 2016.

The study samples comprised 132 Saudi children (76 Male\& 56 female) clinically diagnosed as type 1 diabetes mellitus according to WHO criteria. ${ }^{15,16}$ Sample size was calculated from the simple formula $n=Z^{2} P(1-P) / d^{2}{ }^{17}$ Where, $Z$ is the statistic corresponding to level of confidence, $P$ is expected prevalence ( 0.11 per cent according to some Saudi study), ${ }^{18}$ and $\mathrm{d}$ is precision. In contrast, 72 apparently healthy children (40 males, 32 females) were involved as a control group. Both groups were matched in regard to age and sex.

Exclusion criteria included diabetic children treated with warfarin and heparin, drugs for osteoporosis, 1,25dihydroxy vitamin D3 supplements, glucocorticoids and anticonvulsant drugs. Patients with other types of diabetes mellitus, renal failure, liver disease, anaemia and thyroid disease were also excluded.

An interview with a questionnaire to obtain the clinical data was done for each participant's parent in this study, clinical history and examination of the test group and the controls were done by physicians working in Najran University Hospital \& King Khalid Hospital.

Weight was measured using electronic digital scales. Height was measured using a wall-mounted stadiometer. BMI was subsequently calculated as weight $(\mathrm{kg})$ per height $\left(\mathrm{m}^{2}\right)$.

In sterile condition and using possible antiseptic measurements for skin following an overnight fast (8$12 \mathrm{hrs}), 5 \mathrm{mls}$ of venous blood was collected from each participant and aliquoted into 2 tubes one of which contains EDTA to obtain plasma. For serum preparation, the blood samples were separated after complete clotting by centrifugation at $4,000 \mathrm{rpm}$ for 5 minutes and serum was 
obtained.

From EDTA tubes, HbA1c was measured using chromatography technique (boronate affinity chromatography). The sera were used to measure the concentrations of osteocalcin and vitamin D using direct ELISA (Enzyme-Linked Immunosorbent Assay) from AbcamUK. Fasting plasma glucose (FPG) was measured by using spectrophotometric method.

Results of this study were statistically analysed using statistical package for social science (SPSS) program. Significant differences between groups were assessed by one-way ANOVA and t- test. Correlation matrix was done and the $r$ values were obtained at level of $(p<0.05)$ significance.

\section{Results}

Our study included 132 type 1 diabetic toddlers and schoolage children (76 males and 56 females) admitted to Najran University and King Khalid Hospitals (Najran, Saudi Arabia) and 72 apparently healthy control children (40 males and 32 females), their ages ranged between 4 and 14 years $(10.45 \pm 3.92$ for diabetic group and $10.53 \pm 3.43$ for control group).

Fasting plasma glucose, FPG (mg/dl) was $196.86 \pm 56.32$ (mean \pm S.D.) for diabetic children while it was $82.78 \pm 8.39$ for control group. Results of the studied parameters are listed in Table 1.

Children with type 1 diabetes mellitus were found to have significantly lower BMI than healthy control group (17.46 \pm 1.76 , mean \pm S.D, versus $18.10 \pm 1.42, p<0.009)$.

Our results revealed that $\mathrm{HbA1c}$ per cent was significantly higher in diabetic group than that of healthy control group (9.90 \pm 1.79 vs. $4.53 \pm 0.46, p<0.001)$.

Pearson correlation coefficients (Figure 1) revealed weak negative correlations for FPG with UCOC and TOC in diabetic children $(r=-0.294, \quad p<0.01 \quad$ and $-0.358, \quad p<0.0001$ respectively). Moreover, a weak positive correlation between FPG and BMI was found in our study ( $r=0.212$, $p<0.015)$. Other significant (in terms of $p$ value) correlations were between UCOC and TOC, UCOC and $\mathrm{HbA} 1 \mathrm{C}, \mathrm{BMI}$ and $\mathrm{HbA} 1 \mathrm{C}$, and BMI and TOC $(r=0.865, p<0.0001, r=-0.182$, $p<0.037, \quad r=0.318, \quad p<0.0001$ and $r=-0.206, p<0.019$ respectively).
In healthy control group, Pearson correlation coefficients showed weakly significant negative correlations for BMI with 25(OH)D3, UCOC and TOC ( $r=-0.369, p<0.001, r=-0.205$, $p<0.05$, and $r=-0.239, p<0.043$ respectively) and a moderately highly significant positive correlation for BMI with FPG ( $r=0.411, p<0.0001)$ and significantly negative correlation between 25(OH)D3 and FPG ( $r=-0.62, p<0.0001)$. Strikingly, there was a moderately significant positive correlation between levels of vitamin D and UCOC in healthy control group $(r=0.457, p<0.0001)$. Such significance was not present in diabetic group $(r=-0.077, p<0.38)$.

\section{Discussion}

It has recently reported that the sympathetic tone into osteoblast is a substantial mediator of leptin regulation of insulin secretion by streamlining osteocalcin bioactivity. This unanticipated functional cross talk between nervous systems, fat, and skeleton explains the importance of the skeleton for the regulation of one of the major physiological functions such as glucose homeostasis in vertebrates. ${ }^{19}$

Cell-based studies in experimental animals using isolated pancreatic islets, a beta cell line, and primary adipocytes showed that picomolar amounts of osteocalcin are sufficient to regulate the expression of the insulin genes and beta cell proliferation markers, whereas nanomolar amounts affect adiponectin expression in white and brown adipocytes, respectively. ${ }^{20}$

Furthermore, human studies demonstrated the positive association between insulin secretion, insulin sensitivity and serum osteocalcin levels. ${ }^{21,22}$

In concordance with these reports, our study revealed the weakly significant negative correlations between UCOC and TOC with glucose intolerance represented with FPG in type 1 diabetic children( $r=-0.294, p<0.01$ and $-0.358, p<0.0001$ respectively). Pittas and his colleagues reported in their cross-sectional analyses that serum osteocalcin concentration was inversely associated with fasting plasma glucose $(P=0.01)$, fasting insulin $(P=0.006)$, homeostasis model assessment for insulin resistance $(P=0.002)$, highsensitivity $C$-reactive protein $(P=0.01), I L-6 \quad(P=0.02), B M I$ $(p<0.001)$, and body fat $(p<0.001) .^{23}$ Thus our results support previous studies with regard to the role of osteocalcin in the regulation of glucose tolerance and insulin secretion and sensitivity. There is some debate regarding which form is the active one with respect to these effects. In vitro data and earlier animal studies, as well as some human studies suggested that only the undercarboxylated form of osteocalcin (UCOC) functions 
hormonally in the regulation of glucose homeostasis and energy metabolism, leading to the suggestion that vitamin D promotes insulin production and sensitivity by stimulating UCOC secretion by osteoblasts. ${ }^{24,25}$ Contrarily, Bahijri et al. reported that caboxylated $O C$ is most likely the active form. $^{26}$

BMI showed statistically significant lower values in type 1 diabetic children in comparison to healthy controls. In addition, BMI showed weakly significant negative correlation with total osteocalcin in diabetic and healthy groups $(r=-0.206, \quad p<0.019$ and $r=-0.239, \quad p<0.043$ respectively) suggesting an inefficacious attempt of osteoblasts to compensate for the increased bone resorption. As far as osteocalcin indicates osteoblastic function, in patients with diabetes the bone formation proves to be inefficient enough to compensate for the bone resorption. In agreement with our results, Neumann et al. reported that in T1DM, total OC was inversely correlated with $\mathrm{BMI}$ and $\mathrm{HbA1C}$, and $\mathrm{UCOC}$ inversely correlated with HbA1c. ${ }^{27}$ In diabetes, bone turnover is characterized with osteoblastic insufficiency and hence impaired bone formation is not capable to compensate for normal or increased bone resorption. The suggested reason for this is the insufficiency of insulin and insulin-like growth factors. ${ }^{28,29}$ All these events lead to decreased bone mineral density which negatively affect BMI in addition to the fact that T1DM is initially associated with decreased weight. However, the impact of hyperglycaemia on osteoblast cannot be ignored. Data from in vivo and in vitro studies showed that high glucose deteriorates osteoblast function by causing intracellular assemblage of sorbitol or by generating higher concentration of advanced glycation end products (AGEs) in collagen that may reduce bone density. ${ }^{30,31}$

Our results showed that serum levels of $25(\mathrm{OH}) \mathrm{D} 3$, TOC and UCOC in type 1 diabetic children were severely deficient when compared with that in healthy control children. Vitamin $D$ deficiency has been associated with type 1 diabetes. ${ }^{32}$ The derivation for the divergent roles of vitamin $D$ include the presence of specific vitamin $D$ receptors (VDRs) on pancreatic $\beta$-cells ${ }^{33}$ and skeletal muscle, ${ }^{34}$ the presence of a vitamin $D$ response element in the human insulin gene promoter, ${ }^{35}$ and the expression of $1 \alpha-$ hydroxylase enzyme in pancreatic $\beta$-cells which catalyses the conversion of 25(OH)D to 1,25 -dihydroxyvitamin $D^{36}$ Moreover, Vitamin $D$ attenuates the expression of proinflammatory cytokines involved in insulin resistance such as interleukins IL-1, IL-6, TNF-a, also down regulates NF-Kb (Nuclear factor) activity. ${ }^{37}$ Consequently, vitamin D may have a protective effects on diabetes ${ }^{38,39}$ through its impact on calcium and phosphorus metabolism and regulation of the insulin receptor gene. ${ }^{40}$ In general, largescale population cross-sectional studies have revealed a significant positive relationship between serum $25(\mathrm{OH}) \mathrm{D}$ and measures of insulin sensitivity ${ }^{41,42}$ and that vitamin $D$ is inversely associated with insulin resistance. ${ }^{43}$

Ou et al. observed stronger associations of serum 25OHD with insulin sensitivity in overweight than normal weight subjects, suggesting that overweight subjects with hypovitaminosis $D$ may benefit more from vitamin $D$ replacement than normal weight subjects. ${ }^{44}$

Regarding the correlation between UCOC and $\mathrm{HbA} 1 \mathrm{C}$ in children with type 1 diabetes mellitus (T1DM), our study displayed a significant negative correlation $(r=-0.182$, $p<0.037)$ between these parameters indicating the lower UCOC, the worse glycaemic control in children with T1DM. In addition, as obtained in healthy control group, there were moderately to weakly significant uphill correlations between levels of 25(OH)D3 with UCOC and 25(OH)D3 with FPG $(r=0.457, p<0.0001$ and $r=0.371, p<0.001$ respectively). Previous studies have identified that the deficiency of vitamin $D$ is strongly connected with diabetes mellitus type $1^{45,46}$ and that the death of islet cells and its complements can be easily avoided by the vitamin $D$, which in turn, will promote the production of insulin. ${ }^{47,48}$ Hence, beta cells can be negatively implicated by the lower level of 25(OH)D3. ${ }^{49}$

A comprehensive study was conducted by some investigators in Saudi Arab. They discussed the relationship between deficiency of vitamin $D$ and diabetes mellitus. The results of their study showed more or less the same results as revealed in our study. The mean levels of $250 \mathrm{HD}$ were significantly lower in the T1DM children compared to the controls $(36.7 \pm 14.3 \mathrm{nmol} / \mathrm{l}$ versus $44.8 \pm 14.1 \mathrm{nmol} / \mathrm{I}) .^{50}$

Although many studies pointed out that vitamin $D$ deficiency could be a possible risk factor for diabetes, ${ }^{51,52}$ there is still debate about whether vitamin D deficiency is a sequel of diabetes or a predisposing factor. Many previous studies supported our results that showed the significant decrease in TOC and UCOC as well as the negative correlation between osteocalcin and glycated haemoglobin in diabetic patients. Abo-El-Asrar et al. reported that serum osteocalcin was significantly lower in diabetic patients compared to control group $(p=0.00)^{53}$ while, Pittas et al. found that serum osteocalcin level was inversely correlated with FPG $(p=0.01)$, fasting insulin $(p=0.006)$, homeostasis model assessment for insulin resistance $(p=0.002)$, high- 
sensitivity C-reactive protein $(p=0.01), I L-6(p=0.02), B M I$ $(p<0.001)$, and body fat $(p<0.001) .{ }^{23}$ Similarly, Takaya et al. ${ }^{10}$ reported that serum level of under-carboxylated osteocalcin was negatively correlated with mean blood glucose levels ( $r=-0.447, p=0.013$ ) and glycosylated haemoglobin (HbA1c) $(r=-0.455, p=0.012)$ in diabetic patients. Moreover, Khoshhal et al. detected significantly lower levels of procollagen $\mathrm{N}$ terminal peptide and osteocalcin in children with T1DM. ${ }^{54}$

To our knowledge, it is the first study tried to explore the possible role of circulating osteocalcin in the pathogenesis of T1DM and its correlation with fasting plasma glucose and vitamin D levels in toddlers and schoolchildren in Najran region, Southwestern Saudi Arabia. Nevertheless, our study has some limitations as it was conducted in just 2 hospitals and did not include children with type 2 diabetes mellitus.

\section{Conclusion}

It is obvious that the levels of 25(OH)D3, TOC, UCOC are significantly diminished in children with T1DM suggesting the bidirectional influence of $\beta$ cells on vitamin $D$ and osteocalcin and vice versa. There are significant negative correlations between UCOC and TOC with glucose intolerance represented with FPG in type 1 diabetic children. The reverse (negative) correlation between UCOC and $\mathrm{HbA} 1 \mathrm{c}$ may indicate the probable prognostic value of UCOC as the lower UCOC, the worse glycaemic control in children with T1DM. In addition, there were moderately significant positive correlations between levels of 25(OH)D3 with UCOC and 25(OH)D3 with FPG as well as a significantly negative correlation between 25(OH)D3 and FPG in healthy control group. Therefore, convenient vitamin D supplementation for children could help to counter the growing trend in the pathogenesis of T1DM.

\section{References}

1. Hassan MM, Alashmawy AA, Sharaf SA, et al. Vitamin D status in Egyptian children and adolescents with type 1 diabetes mellitus. J Diabetes Metab. 2016;6:636. doi:10.4172/2155-6156.1000636.

2. Saki F, Omrani GR, Pouralborz $Y$, et al. Vitamin D deficiency and the associated factors in children with type 1 diabetes mellitus in southern Iran. Int J Diabetes Dev Ctries. 2016;37(1):78-84. doi:10.1007/s13410-0160499-0.

3. Bloomgarden ZT. Type 2 diabetes in the young. Diabetes Care. 2004;27:998-1010. doi:10.2337/diacare.27.4.998.

4. Hummel S, Hummel M, Banholzer J, et al. Development of autoimmunity to transglutaminase $C$ in children of patients with type 1 diabetes: relationship to islet autoantibodies and infant feeding. Diabetologia.
2007;50:390-394.

5. Al-Agha $A E$, Alafif $M$, Abd-Elhameed IA. Glycemic control, complications, and associated autoimmune diseases in children and adolescents with type 1 diabetes in Jeddah, Saudi Arabia. Saudi Med J. 2015;36(1):26-31. doi: 10.15537/smj.2015.1.9829.

6. Sayed MH, Hegazi MA, Abdulwahed K, et al. Risk factors and predictors of uncontrolled hyperglycemia and diabetic ketoacidosis in children and adolescents with type 1 diabetes mellitus in Jeddah, western Saudi Arabia. J Diabetes. 2017;9(2):190-199. doi: 10.1111/17530407.12404.

7. Al-Rubean K. National surveillance for type 1, type 2 diabetes and prediabetes among children and adolescents: a population-based study (SAUDI-DM). J Epidemiol Community Health. 2015;69:1045-1051. doi:10.1136/jech-2015-205710.

8. American Diabetes Association. 11. Children and Adolescents. Diabetes Care. 2016;39:S86-S93. http://dx.doi.org/10.2337/dc16-S014.

9. Al Shaikh A, Al Zahrani AM. Impact of Vitamin D Status on Cardiometabolic Complications among Children and Adolescents with Type 1 Diabetes Mellitus. J Clin Res Pediatr Endocrinol. 2016;8:48-54.

10. Takaya J, Tanabe Y, Kuroyanagi Y, et al. Decreased undercarboxylated osteocalcin in children with type 2 diabetes mellitus. J Pediatr Endocrinol Metab. 2016;29(8):879-884.

11. Sultan E, Taha I, Saber LM. Altered Bone Metabolic Markers In Type 2 Diabetes Mellitus: Impact of Glycemic Control. J Taibah Univ Med Sci. 2008;3(2):104-116.

12. Chau DL, Edelman SV. Osteoporosis and Diabetes. Clin Diabetes. 2002;20:153-157.

13. Uitterlinden AG, Fang Y, Van Meurs JB, et al. Genetics and biology of vitamin $D$ receptor polymorphisms. Gene. 2004;338:143-156.

14. Nakashima A, Yokoyama K, Yokoo T, et al. Role of vitamin $\mathrm{D}$ in diabetes mellitus and chronic kidney disease. World J Diabetes. 2016;7(5):89-100. doi:10.4239/wjd.v7.i5.89.

15. World Health Organization: Definition and diagnosis of diabetes mellitusand intermediate hyperglycemia. Report of WHO/IDF consultation, 2006.

16. American Diabetes Association. Classification and diagnosis of diabetes. Diabetes Care. 2015;38:S8-S16. http://dx.doi.org/10.2337/dc15-S005.

17. Pourhoseingholi MA, Vahedi M, Rahimzadeh M. Sample size calculation in medical studies. Gastroenterol Hepatol Bed Bench. 2013;6:14-17.

18. Al-Herbish AS, El-Mouzan MI, Al-Salloum AA, et al. Prevalence of type 1 diabetes mellitus in Saudi Arabian 
children and adolescents. Saudi Med J. 2008;29:12851288.

19. Hinoi E. Pivotal role of skeletal tissues in the regulation mechanisms for physiological functions mediated by multiple organ networks. Yakugaku Zasshi. 2012;132(6):721-5.

20. Ferron M, Hinoi E, Karsenty $G$, et al. Osteocalcin differentially regulates beta cell and adipocyte gene expression and affects the development of metabolic diseases in wild-type mice. Proc Natl Acad Sci U S A. 2008;105(13):5266-70. doi: 10.1073/pnas.0711119105.

21. Fernández-Real JM, Izquierdo $M$, Ortega $F$, et al. The relationship of serum osteocalcin concentration to insulin secretion, sensitivity, and disposal with hypocaloric diet and resistance training. J Clin Endocrinol Metab. 2009;94(1):237-245.

22. Hwang YC, Jeong IK, Ahn KJ, et al. Circulating osteocalcin level is associated with improved glucose tolerance, insulin secretion and sensitivity independent of the plasma adiponectin level. Osteoporos Int. 2012;23(4):1337-1342.

23. Pittas AG, Harris SS, Eliades $M$, et al. Association between serum osteocalcin and markers of metabolic phenotype. J Clin Endocrinol Metab. 2009;94(3):827-32. doi: 10.1210/jc.2008-1422.

24. Hwang YC, Jeong IK, Ahn KJ, et al. The uncarboxylated form of osteocalcin is associated with improved glucose tolerance and enhanced $\beta$-cell function in middle-aged male subjects. Diabetes Metab Res Rev. 2009;25(8):768772.

25. Wu YY, Yu T, Zhang XH, et al. 1,25(OH)2D3 inhibits the deleterious effects induced by high glucose on osteoblasts through undercarboxylated osteocalcin and insulin signaling. J Steroid Biochem Mol Biol. 2012;132(12):112-119.

26. Bahijri SM, Qusti S, Khormi F, et al. Carboxylated osteocalcin and adiponectin correlate with glycated hemoglobin and $25(\mathrm{OH})$ vitamin $D$ levels in Saudi females with type 2 diabetes mellitus following ingestion of vitamin $\mathrm{D}$ supplements. $\mathrm{Br} J$ Med Med Res. 2015;6(4):404-414.

27. Neumann T, Lodes S, Kästner B, et al. Osteocalcin, adipokines and their associations with glucose metabolism in type 1 diabetes. Bone. 2016;82:50-5. doi: 10.1016/j.bone.2015.04.017.

28. Rico H, Hernandez ER, Cabranes JA, et al. Suggestion of a deficient osteoblastic function in diabetes mellitus: the possible cause of osteopenia in diabetics. Calcif Tissue Int. 1989;45:71-73.

29. Botushanov N, Yaneva M, Orbetzova $M$, et al. Bone Mineral Density in Bulgarian Patients with Type 1
Diabetes Mellitus. J Osteopor Phys Act. 2014;2:113. doi:10.4172/2329-9509.1000113.

30. Inaba M, Terada M, Nishizawa $Y$, et al. Protective effect of an aldose reductase inhibitor against bone loss in galactose-fed rats: possible involvement of the polyol pathway in bone metabolism. Metabolism. 1999;48:904-909.

31. Saito M, Fujii K, Mori Y, et al. Role of collagen enzymatic and glycation induced cross-links as a determinant of bone quality in spontaneously diabetic WBN/Kob rats. Osteoporos Int. 2006;17:1514-1523.

32. Mathieu C, Gysemans C, Giulietti A, et al. Vitamin D and diabetes. Diabetologia. 2005;48:1247-1257.

33. Johnson JA, Grande JP, Roche PC, et al. Immunohistochemical localization of the 1,25(OH)2D3 receptor and calbindin D28k in human and rat pancreas. Am J Physiol. 1994;267(3):E356-E360.

34. Simpson RU, Thomas GA, Arnold AJ. Identification of 1, 25-dihydroxyvitamin D3 receptors and activities in muscle. J Biol Chem. 1985;260(15):8882-91.

35. Maestro B, Davila N, Carranza MC, et al. Identification of a Vitamin $D$ response element in the human insulin receptor gene promoter. J Steroid Biochem Mol Biol. 2003;84(2-3):223-230.

36. Bland R, Markovic D, Hills CE, et al. Expression of 25hydroxyvitamin D3-1 $\alpha$-hydroxylase in pancreatic islets. $J$ Steroid Biochem Mol Biol. 2004;89-90:121-125.

37. Cohen-Lahav M, Douvdevani A, Chaimovitz $C$, et al. The anti-inflammatory activity of 1,25dihydroxy vitamin D3 in macrophages. J Steroid Biochem Mol Biol. 2007;103:558-562. doi: 10.1016/j.jsbmb.2006.12.093.

38. Talaei A, Mohamadi $M$, Adgi Z. The effect of vitamin D on insulin resistance in patients with type 2 diabetes. Diabetol Metab Syndr. 2013;5(8):1-5. doi:10.1186/17585996-5-8.

39. Mohammadi SM, Eghbali SA, Soheilikhah S, et al. The effects of vitamin $D$ supplementation on adiponectin level and insulin resistance in first-degree relatives of subjects with type 2 diabetes: a randomized doubleblinded controlled trial. Electron Physician. 2016;8(9):2849-2854.

40. Maestro B, Molero S, Bajo S, et al. Transcriptional activation of the human insulin receptorgene by 1,25 dihydroxyvitamin $\mathrm{D}(3)$. Cell Biochem Funct. 2002;20:227-232.

41. Scragg R, Sowers M, Bell C. Serum 25-hydroxyvitamin D, diabetes, and ethnicity in the Third National Health and Nutrition Examination Survey. Diabetes care. 2004;27(12):2813-8.

42. Kamycheva E, Jorde R, Figenschau $\mathrm{Y}$, et al. Insulin sensitivity in subjects with secondary 
hyperparathyroidism and the effect of a low serum 25hydroxyvitamin D level on insulin sensitivity. J Endocrinol Invest. 2007;30(2):126-32.

43. Alvarez JA, Ashraf A. Role of Vitamin D in Insulin Secretion and Insulin Sensitivity for Glucose Homeostasis. Int J Endocrinol. 2010;2010:351385. http://dx.doi.org/10.1155/2010/351385.

44. Ou, HY, Karnchanasorn R, Lee LZ, et al. Interaction of BMI with vitamin $D$ and insulin sensitivity. Eur J Clin Invest. 2011;41:1195-1201. doi:10.1111/j.13652362.2011.02525.x.

45. Al-Agha AE, Ahmad IA. Association among Vitamin D Deficiency, Type 1 Diabetes Mellitus and Glycemic Control. J Diabetes Metab. 2015;6:594. doi:10.4172/2155-6156.1000594.

46. Faienza MF, Ventura $A$, Delvecchio $M$, et al. High Sclerostin and Dickkopf-1 (DKK-1) serum levels in children and adolescents with type 1 diabetes mellitus. J Clin Endocrinol Metab. 2016. doi: 10.1210/jc.2016-237.

47. Holick MF, Binkley NC, Bischoff-Ferrari HA, et al. Evaluation, treatment, and prevention of vitamin $D$ deficiency: an Endocrine Society clinical practice guideline. J Clin Endocrinol Metab. 2011;96:1911-1930.

48. Janner $M$, Ballinari $P$, Mullis $P E$, et al. High prevalence of vitamin $D$ deficiency in children and adolescents with type 1 diabetes. Swiss Med Wkly. 2010;140:w13091.

49. Pozzilli P, Manfrini S, Crinò A, et al. Low levels of 25hydroxyvitamin D3 and 1,25-dihydroxyvitamin D3 in patients with newly diagnosed type 1 diabetes. Horm Metab Res. 2005;37:680-683.

50. Bin-Abbas BS, Jabari MA, Issa SD, et al. Vitamin D levels in Saudi children with type 1 diabetes. Saudi Med J. 2011;32:589-592.

51. Ye Z, Sharp SJ, Burgess S, et al. Association between circulating 25-hydroxyvitamin $\mathrm{d}$ and incident type 2 diabetes: A mendelianrandomisation study. Lancet Diabetes Endocrinol. 2015;3(1):35-42.

52. Badenhoop K. Genetics: Vitamin d and type 2 diabetes mellitus--hype or hope? Nat Rev Endocrinol. 2015;11(1):10-11. doi: 10.1038/nrendo.2014.206.

53. Abo-El-Asrar M, Farid SM, Maraghy MOE, et al. Serum Osteocalcin, Zinc Nutritive Status and Bone Turnover in Children and Adolescents with Type1 Diabetes Mellitus. J Diabetes Metab. 2011;2:128. doi:10.4172/21556156.1000128.

54. Khoshhal KI, Sheweita S, Al-Maghamsi MS, et al. Dose type 1 diabetes mellitus affect bone quality in prepubertal children? J Taibah Univ Med Sci. 2015;10:300-305.

\section{ACKNOWLEDGEMENTS}

This work was supported by a grant from the Deanship of Scientific Research, Najran University, Saudi Arabia (NU/MID/14/035).

\section{PEER REVIEW}

Not commissioned. Externally peer reviewed.

\section{CONFLICTS OF INTEREST}

The authors declare that they have no competing interests.

\section{FUNDING}

Deanship of Scientific Research, Najran University, Saudi Arabia (NU/MID/14/035)

\section{ETHICS COMMITTEE APPROVAL}

All patients and their parents were assured that all their obtained information will be handled in a confidential atmosphere and it will not affect their life after taking verbal and written consent. Ethical clearance and protocol approval were obtained from research and ethics committee of Najran University, Saudi Arabia. All procedures followed were in accordance with the ethical standards of the responsible committee on human experimentation (institutional and national) and with the Helsinki Declaration of 1975, as revised in Brazil 2013. 
Table 1: Results of the study

\begin{tabular}{|l|l|l|}
\hline Parameters & Type 1 Diabetic children & Healthy control children \\
\hline Vit D (nglml) & $10.18 \pm 6.31^{* *}$ & $69.25 \pm 21.44$ \\
\hline UCOC $(\mathrm{ng} / \mathrm{ml})$ & $1.08 \pm 0.56^{* *}$ & $2.578 \pm 0.55$ \\
\hline TOC $(\mathrm{ng} / \mathrm{ml})$ & $2.42 \pm 1.36^{* *}$ & $14.10 \pm 1.52$ \\
\hline FPG $(\mathrm{mg} / \mathrm{dl})$ & $196.86 \pm 56.32^{* *}$ & $82.78 \pm 8.39$ \\
\hline HbA1c \% & $9.90 \pm 1.79^{* *}$ & $4.53 \pm 0.46$ \\
\hline
\end{tabular}

Data are expressed as means \pm S.D

Vit D: 25- (OH) vitamin D3; UCOC: under-carboxylated osteocalcin; TOC: total osteocalcin; FPG: fasting plasma glucose; HbA1c: glycated haemoglobin

** Highly significant $(p<0.001)$

Figure 1: Scatter plots explain the correlations between FPG and TOC, FPG and UCOC, FPG and 25(OH)D3 as well as FPG and $\mathrm{BMI}$ in type 1 diabetic children and healthy control groups
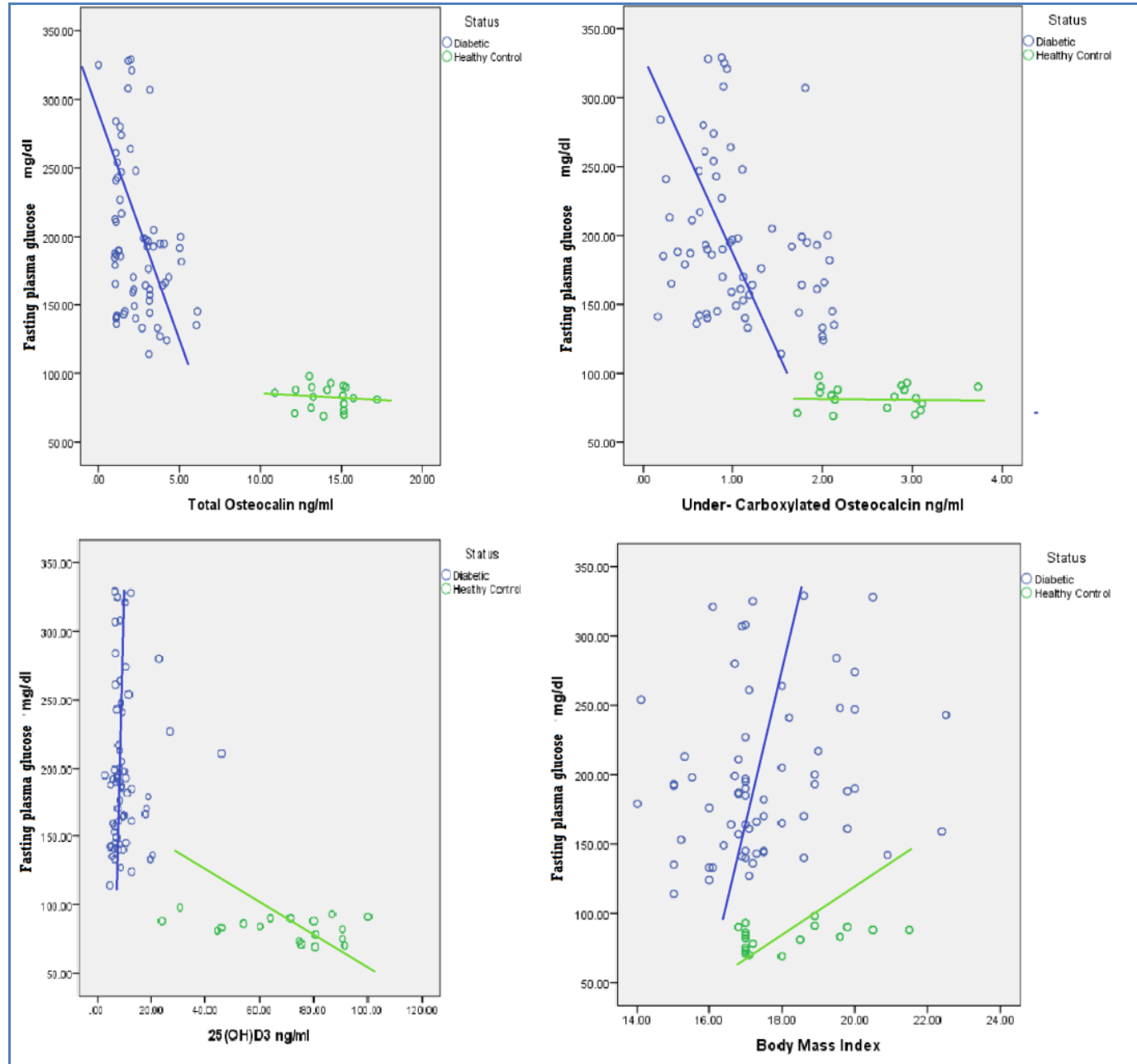